\title{
6.2 GENERAL RELATIVISTIC THEORY OF ROTATING NEUTRON STARS - A REVIEW
}

\author{
JEFFREY M. COHEN * \\ Institute for Advanced Study, Princeton, N.J., U.S.A.
}

Except in cosmology, astrophysicists are used to thinking of general relativistic effects as small (e.g., light bending, perihelion advance, red shift) and have generally left such problems to general relativists. However, the discovery of pulsars (Hewish et al., 1968) may have changed this. Not only is general relativity necessary to treat rotating neutron stars, but relativity was also partly responsible for the elimination of pulsating white dwarfs as pulsar models.

Soon after the discovery of pulsars, pulsating white dwarfs were suggested as pulsar models. The idea was that, if the white dwarf were composed of a material with a high electron capture threshold (e.g. $13.5 \mathrm{MeV}$ for $\mathrm{C}^{12}$ ), the fundamental pulsation period might correspond to the pulsar periods. In Newtonian theory, the pulsation period decreases with increasing central density when there is no electron capture. However, when general relativistic effects were accounted for, (Skilling, 1968; Faulkner and Cribben, 1968; Cohen, 1968c) it was found that the pulsation reached a minimum and then increased again - the minimum period being larger than $1.33 \mathrm{sec}$. The reason for this general relativistic instability is that stars become unstable when the adiabatic index becomes $\left(\frac{4}{3}\right)+\varepsilon, \varepsilon>0$. With the discovery of the Crab pulsar, all hope was abandoned of describing pulsars as pulsating white dwarfs.

It is presently believed that pulsars are rotating neutron stars (Gold, 1968). Because of their high density, rotating neutron stars must be treated via general relativity. Such a treatment is much more complicated than the corresponding Newtonian problem. In particular new effects arise such as the dragging along of inertial frames by rotating masses. The general relativistic equations describing a rotating neutron star are the following (Cohen and Brill, 1968)

$$
\begin{aligned}
-8 \pi T^{00}= & B^{-1} C^{-1}\left[\left(C_{r} / B\right)_{r}+\left(B_{\theta} / C\right)_{\theta}+E^{-1}\left(C E_{r} / B\right)_{r}\right. \\
& \left.+E^{-1}\left(B E_{\theta} / C\right)_{\theta}\right]+\left(E \Omega_{r} / 2 A B\right)^{2}+\left(E \Omega_{\theta} / 2 A C\right)^{2} \\
-8 \pi\left(T^{11}-\right. & \left.\frac{1}{2} T\right)=B^{-1} C^{-1}\left[\left(C_{r} / B\right)_{r}+\left(B_{\theta} / C\right)_{\theta}\right] \\
& +B^{-1} E^{-1}\left[\left(E_{r} / B\right)_{r}+\left(B_{\theta} E_{\theta} / C^{2}\right)\right]+A^{-1} B^{-1}\left[\left(A_{r} / B\right)_{r}\right. \\
& \left.+\left(A_{\theta} B_{\theta} / C^{2}\right)\right]-\frac{1}{2}\left(E \Omega_{r} / A B\right)^{2} \\
-8 \pi\left(T^{22}-\right. & \left.\frac{1}{2} T\right)=B^{-1} C^{-1}\left[\left(C_{r} / B\right)_{r}+\left(B_{\theta} / C\right)_{\theta}\right] \\
& +C^{-1} E^{-1}\left[\left(E_{\theta} / C\right)_{\theta}+\left(E_{r} C_{r} / B^{2}\right)\right]+A^{-1} C^{-1}\left[\left(A_{\theta} / C\right)_{\theta}\right. \\
& \left.+\left(A_{r} C_{r} / B^{2}\right)\right]-\frac{1}{2}\left(E \Omega_{\theta} / A C\right)^{2}
\end{aligned}
$$

\footnotetext{
* This work was supported in part by the US Air Force Office of Scientific Research, Office of Aerospace Research under AFOSR Grant 70-1866.
} 


$$
\begin{aligned}
&-8 \pi\left(T^{33}-\right.\left.\frac{1}{2} T\right)=B^{-1} E^{-1}\left[\left(E_{r} / B\right)_{r}+\left(B_{\theta} E_{\theta} / C^{2}\right)\right] \\
&+C^{-1} E^{-1}\left[\left(E_{\theta} / C\right)_{\theta}+\left(E_{r} C_{r} / B^{2}\right)\right]+A^{-1} E^{-1}\left[\left(A_{r} E_{r} / B^{2}\right)\right. \\
&\left.+\left(A_{\theta} E_{\theta} / C^{2}\right)\right]+\frac{1}{2}\left(E \Omega_{\theta} / A C\right)^{2}+\frac{1}{2}\left(E \Omega_{r} / A B\right)^{2} \\
&-8 \pi T^{12}= C^{-1} E^{-1}\left[\left(E_{r} / B\right)_{\theta}-\left(E_{\theta} C_{r} / B C\right)\right] \\
&+A^{-1} C^{-1}\left[\left(A_{r} / B\right)_{\theta}-\left(A_{\theta} C_{r} / B C\right)\right]-\left(E^{2} \Omega_{r} \Omega_{\theta} / 2 A^{2} B C\right) \\
&-16 \pi B C E^{2} T^{03}=\left(C E^{3} \Omega_{r} / A B\right)_{r}+\left(B E^{3} \Omega_{\theta} / A C\right)_{\theta} .
\end{aligned}
$$

where the quantities $A, B, C, E, \Omega$ are components of the metric

$$
\mathrm{d} s^{2}=-A^{2} \mathrm{~d} t^{2}+B^{2} \mathrm{~d} r^{2}+C^{2} \mathrm{~d} \theta^{2}+E^{2}(\mathrm{~d} \varphi-\Omega \mathrm{d} t)^{2}
$$

and where the components of the stress-energy tensor are given by

$$
T^{\mu \nu}=\left(\varrho+p c^{-2}\right) U^{\mu} U^{\nu}+\eta^{\mu \nu} p c^{-2} .
$$

In the metric (2), $\mathrm{d} s$ represents the distance between two nearby points. Although this set of equations represents a reduction from 10 non-linear equations to 6 it is still rather complicated. But they are valid even for strong gravitational fields and rapid rotation.

Fortunately, a simpler method is available for treating rotating neutron stars. This method (Brill and Cohen, 1966; Cohen and Brill, 1968; Cohen, 1965, 1967, 1968a), which assumes only slow rotation, is valid even for strong gravitational fields. It allows a fully relativistic treatment of rotating stellar models and has been applied to such problems by various authors, e.g. Cohen and Brill (1968), Hartle and Thorne (1968), Cohen and Cameron (1969).

At first sight one might think that a star, such as the Crab pulsar, rotating thirty times a second, is not slowly rotating. However, for a typical neutron star of radius $13 \mathrm{~km}$, mass $1.3 \times 10^{33} \mathrm{gm}$, and rotational period $33 \mathrm{msec}$ or more, the conditions for slow rotation are fulfilled. These conditions are that the 'centrifugal' force acting on any element of the star be small compared to the gravitational force and that the velocity of any element be small relative to the light velocity.

Once the non-rotating models are known, only one equation needs to be integrated in order to describe a slowly rotating neutron star. The non-rotating models are obtained by integrating the equations

$$
\begin{aligned}
m_{r} & =4 \pi r^{2} \varrho \\
c^{2} \varphi_{r} & =G m r^{-2}\left[\frac{1+4 \pi r^{3} p m^{-1} c^{-2}}{1-2 G m r^{-1} c^{-2}}\right], \\
p_{r} & =-\varrho c^{2} \varphi_{r}\left[1+p \varrho^{-1} c^{-2}\right] .
\end{aligned}
$$

Here $m$ is the mass, $\varrho$ the density, $p$ the pressure, $r$ the radius, $c$ the light speed, $G$ the gravitational constant, and $\varphi$ the 'gravitational potential'. These equations are very similar to the corresponding Newtonian equations which can be obtained by setting the expression in brackets equal to 1 . Using an improved equation of state, these 
equations have been applied to obtain neutron star models (Cohen et al., 1969a; Cohen and Cameron, 1970). I will not discuss these models here since this is being done at this symposium by Cameron (1971). For a discussion of the stability of general relativistic models, see, e.g. Chandrasekhar (1964), Taub (1962), Cohen et al. (1969a), Bardeen et al. (1966), Cocke (1965).

Given the non-rotating equilibrium model, one can describe a slowly rotating neutron star by integrating the single equation (Brill and Cohen, 1966; Cohen and Brill, 1968; Cohen, 1968a).

$$
\left[A^{-1} B^{-1} r^{4} \Omega_{r}\right]_{r}=-16 \pi B A^{-1}\left(\varrho+p c^{-2}\right)(\omega-\Omega) G c^{-2} .
$$

Here $A$ and $B$ are given

$$
A=e^{\varphi}, \quad B=1-2 G m r^{-1} c^{-2},
$$

$\omega(r)$ is the angular velocity of the star, and $\Omega$ is the angular velocity of inertial frames along the rotation axis. In general relativity, as in Newtonian mechanics, an inertial frame has the property that 'Coriolis' and 'centrifugal' forces vanish in this frame. However, unlike in Newtonian mechanics, inertial frames in the vicinity of a rotating star are dragged along by the star and rotate relative to those far from the star. The angular velocity of inertial frames can be determined, e.g., by measuring the angular velocity of the axis of a gyroscope (Brill and Cohen, 1966, Cohen and Cohen, 1969).

Using the above results, it is easy to compute quantities of astrophysical interest such as the angular momentum $J$ and rotational energy $E_{\text {rot }}$ of the star. The latter quantity is quite useful for determining, e.g., a lower limit on the mass of a pulsar as can be done for the Crab pulsar (Cohen and Cameron, 1969).

As is well known in quantum mechanics and Newtonian mechanics, symmetries give rise to conserved quantities. For example, momentum and energy conservation are consequences of space and time translation invariance while angular momentum conservation is a consequence of rotational invariance. For a slowly rotating star, the angular momentum is given by

$$
J=\int_{0}^{R} \mathrm{~d} r \mathrm{~d} \theta \mathrm{d} \varphi \sin ^{3} \theta \varrho r^{4} \omega\left[\left(1+p \varrho^{-1} c^{-2}\right) B A^{-1}\left(1-\Omega \omega^{-1}\right)\right]
$$

where $R$ is the radius of the star. This expression (6) differs from the corresponding Newtonian expression by the quantity in brackets. Note that the pressure $p$ as well as the density $\varrho$ contributes to the angular momentum. Also, the motion of inertial frames, and the red shift $\left(z=e^{-\phi}-1=A^{-1}-1\right)$ enter into the general relativistic expression for the angular momentum.

In general relativity, the total energy of a finite size star is equal to its gravitational (Schwarzschild) mass (see, e.g., Landau and Lifshitz, 1962; Møller, 1962). Thus, it 
is reasonable to define the rotational energy of a star to be the total mass of the rotating star minus the mass of the same star when it is not rotating. The rotating and non-rotating stars have the same number of baryons. When we take into account baryon conservation, gravitational red shift, the contribution of space curvature to the volume element, and the motion of inertial frames, we obtain the following positive definite expression for the rotational kinetic energy of a slowly rotating star

$$
\begin{aligned}
E_{\mathrm{rot}}=(4 \pi / 3) \int_{0}^{R} \mathrm{~d} r \varrho r^{4} \omega^{2}\left[\left(1+p \varrho^{-1} c^{-2}\right) B A^{-1}\left(1-\Omega \omega^{-1}\right)^{2}\right] \\
+\int_{0}^{R} \mathrm{~d} r r^{4} \Omega r c^{2}(12 A B G)^{-1}+G J^{2} R^{-3} c^{-2} .
\end{aligned}
$$

If the bracketed factors in the first integral are set equal to 1 and second two terms set equal to zero, the familiar Newtonian expression results. From inspection of Equation (7), it may be interesting to note that the expression for the rotational energy contains contributions from the pressure, red shift, and motion of inertial frames.

Various expressions for the rotational energy can be obtained by, e.g., multiplying Equation (5) by $\Omega$, integrating over all space, and combining the result with Equation (7) yielding

$$
E_{\mathrm{rot}}=(4 \pi / 3) \int_{0}^{R} \mathrm{~d} r \varrho r^{4} \omega^{2}\left[\left(1+p \varrho^{-1} c^{-2}\right) B A^{-1}\left(1-\Omega \omega^{-1}\right)\right] .
$$

For uniform rotation, the expression for the rotational energy takes the simple form

$$
E_{\text {rot }}=J \omega / 2 \text {. }
$$

This result (7b) can be obtained via physical arguments (Zel'dovich, 1970; Thorne, 1970) and via variational principles (Hartle, 1970).

The expression (7b) has exactly the same form as it does in Newtonian mechanics even when $E_{\text {rot }}$ and $J$ contain large general relativistic contributions. That this is reasonable can be seen from the following physical argument: surround the star with a concentric shell of negligible mass and with radius sufficiently large that the shell resides in flat space. If the shell is coupled to the star in such a way that the two rotate together, then we can work with the shell without worrying about what is inside. Since the gravitational field in the vicinity of the shell is weak, a Newtonian treatment can be used to obtain the familiar Newtonian relation (7b).

If the energy source of the Crab nebula is the Crab pulsar (Wheeler, 1966; Finzi and Wolf, 1969), then a lower limit on the mass of the Crab pulsar can be obtained by equating the rate of loss of rotational energy with the observed electromagnetic energy emitted by the $\mathrm{Crab}$. When this is done, with general relativistic effects taken into account, a lower limit of about 0.4 solar masses is obtained (Cohen and Cameron, 1969). 
For completeness, a table of rotational properties of a typical neutron star model is included here. From Table I, one can see that general relativistic effects can be quite large. The ratio of radius to gravitational radius can be as small as about 1.65 and the angular velocity of inertial frames at the center of the star can be as high as about 79 per cent of the angular velocity of the star (Figure 1).

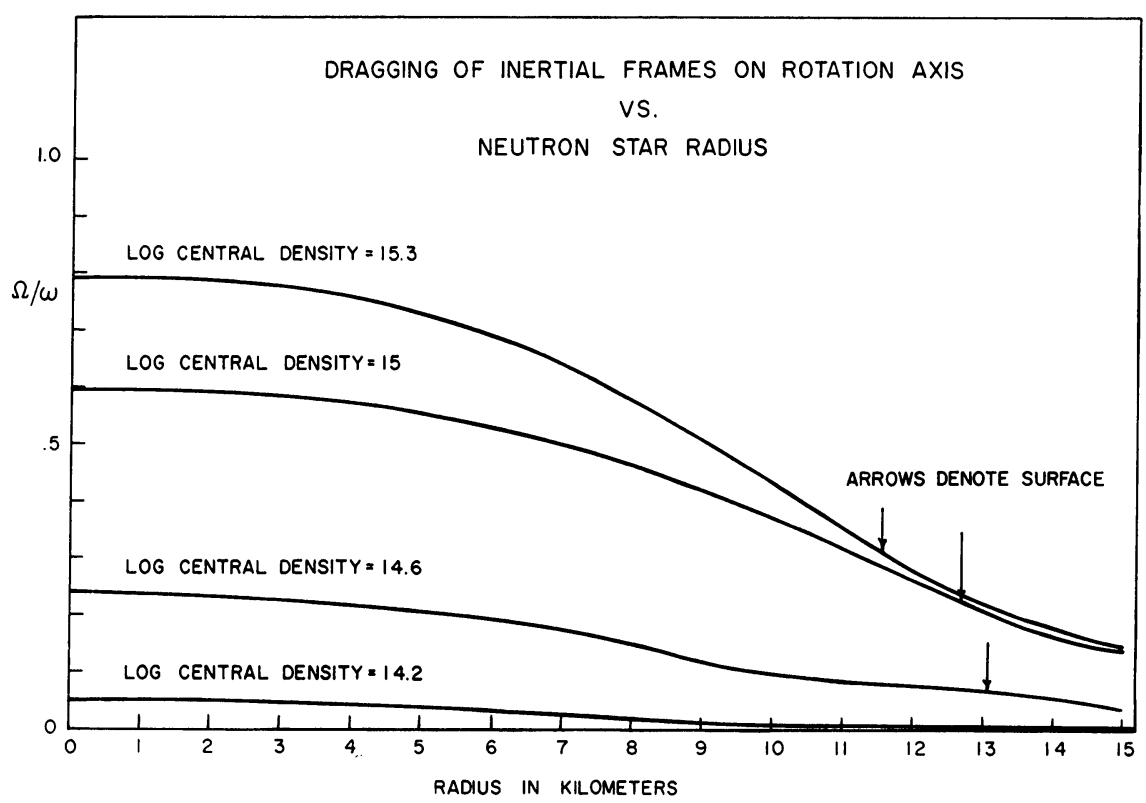

Fig. 1. Dragging along of inertial frames on rotation axis as a function of radius. The angular velocity of inertial frames along the rotation axis is equal to $\Omega$ while $\omega$ is the angular velocity of the neutron star. Both of these angular velocities are measured by an observer in an inertial frame far from the star. Outside the star $\Omega$ is given analytically by $\Omega=2 G J r^{-3} c^{-2}$.

Besides influencing the structure and stability of neutron stars, and giving rise to an induced rotation of inertial frames, general relativity also alters the magnetic field exterior to the neutron star. A frozen-in magnetic field has been postulated by various authors to explain the slowing down and the emission from pulsars (Goldreich and Julian, 1969; Gunn and Ostriker, 1969; Canuto and Chiu, 1968; Michel, 1969). Instead of falling off as $r^{-(2+l)}$ as in flat space, each magnetic multipole varies as a hypergeometric function of radius. In Table II is given the ratio of the magnetic field components in flat space to those which take general relativity into account. The general relativistic contributions become more pronounced as the multipolarity increases. Further details can be found elsewhere (Anderson and Cohen, 1970), see also Ginzburg and Ozernoi (1965) and Cruz et al. (1969) for a discussion of the dipole case.

From the above, it seems that general relativistic effects can be quite important in neutron star theory and should not be neglected. 
TABLE I

Rotational properties of selected neutron star models, $V_{\gamma}$ potential

\begin{tabular}{lllll}
$\begin{array}{l}\text { Log } \\
\text { central } \\
\text { density } \\
\mathrm{gm} / \mathrm{cm}^{3}\end{array}$ & $\begin{array}{l}\text { Radius/ } \\
\text { Gravitational } \\
\text { radius }\end{array}$ & $\begin{array}{l}J / \omega \\
\mathrm{gm} / \mathrm{cm}^{2}\end{array}$ & $\begin{array}{l}\text { Central dragging } \\
\text { of inertial frames } \\
\Omega_{c / \omega}\end{array}$ & $\begin{array}{l}\text { Surface } \\
\text { dragging } \\
\text { of inertial } \\
\text { frames } \\
\Omega_{8 / \omega}\end{array}$ \\
\hline 14 & & & & $1.6 \times 10^{-4}$ \\
14.1 & 498 & $1.93 \times 10^{48}$ & 0.024 & $4.9 \times 10^{-6}$ \\
14.2 & 538 & $3.29 \times 10^{43}$ & 0.028 & $1.1 \times 10^{-3}$ \\
14.3 & 58.7 & $5.48 \times 10^{43}$ & 0.046 & $6.1 \times 10^{-3}$ \\
14.4 & 24.2 & $1.27 \times 10^{44}$ & 0.073 & 0.017 \\
14.5 & 12.9 & $2.78 \times 10^{44}$ & 0.11 & 0.032 \\
14.6 & 8.34 & $5.16 \times 10^{44}$ & 0.16 & 0.054 \\
14.7 & 5.59 & $8.95 \times 10^{44}$ & 0.23 & 0.080 \\
14.8 & 4.13 & $1.37 \times 10^{45}$ & 0.21 & 0.11 \\
14.9 & 3.30 & $1.84 \times 10^{45}$ & 0.39 & 0.14 \\
15 & 2.71 & $2.30 \times 10^{45}$ & 0.47 & 0.18 \\
15.1 & 2.27 & $2.69 \times 10^{45}$ & 0.56 & 0.22 \\
15.2 & 1.99 & $2.94 \times 10^{45}$ & 0.65 & 0.25 \\
15.3 & 1.79 & $3.02 \times 10^{45}$ & 0.72 & 0.29 \\
15.4 & 1.65 & $2.94 \times 10^{45}$ & 0.79 & 0.32 \\
\hline
\end{tabular}

TABLE II

Ratio of curved space magnetic field components to flat space components. Radial ratio is $R_{1}$; transverse ratio is $R_{2}$

\begin{tabular}{|c|c|c|c|c|c|c|c|c|}
\hline$(r / 2 m)-1$ & $l=1$ & & $l=2$ & & $l=3$ & & $l=4$ & \\
\hline & $R_{1}$ & $R_{2}$ & $R_{1}$ & $R_{2}$ & $R_{1}$ & $R_{2}$ & $R_{1}$ & $R_{2}$ \\
\hline $1.0 \times 10^{3}$ & 1.0 & 1.0 & 1.0 & 1.0 & 1.0 & 1.0 & 1.0 & 1.0 \\
\hline $1.0 \times 10^{2}$ & 1.0 & 1.0 & 1.0 & 1.0 & 1.0 & 1.0 & 1.0 & 1.0 \\
\hline $1.0 \times 10$ & 1.1 & 1.1 & 1.0 & 1.0 & 1.2 & 1.2 & 1.3 & 1.3 \\
\hline 5.0 & 1.1 & 1.2 & 1.3 & 1.4 & 1.4 & 1.4 & 1.5 & 1.6 \\
\hline 4.0 & 1.2 & 1.2 & 1.3 & 1.4 & 1.5 & 1.5 & 1.7 & 1.7 \\
\hline 3.0 & 1.2 & 1.3 & 1.4 & 1.5 & 1.7 & 1.7 & 1.9 & 2.0 \\
\hline 2.0 & 1.3 & 1.5 & 1.7 & 1.8 & 2.1 & 2.2 & 2.5 & 2.6 \\
\hline 1.0 & 1.6 & 1.9 & 2.3 & 2.6 & 3.3 & 3.5 & 4.5 & 4.8 \\
\hline $6.0 \times 10^{-1}$ & 2.0 & 2.5 & 3.3 & 3.8 & 5.2 & 6.3 & 8.2 & 8.9 \\
\hline $1.0 \times 10^{-1}$ & 4.0 & 6.7 & 12 & 17 & 28 & 36 & 69 & 85 \\
\hline $1.0 \times 10^{-5}$ & 30 & $9.3 \times 10^{2}$ & $1.7 \times 10^{2}$ & $3.1 \times 10^{3}$ & $38.3 \times 10^{2}$ & $1.1 \times 10^{4}$ & 2.6 & )$^{3} 1.2 \times 10^{4}$ \\
\hline $1.0 \times 10^{-10}$ & 64 & $3.0 \times 10^{5}$ & $4.0 \times 10^{2}$ & $9.7 \times 10^{5}$ & $52.0 \times 10^{3}$ & $3.3 \times 10^{6}$ & $9.5 \times$ & )$^{3} 1.2 \times 10^{7}$ \\
\hline
\end{tabular}

\section{References}

Anderson, J. L. and Cohen, J. M.: 1970, Astrophys. Space Sci. 9, 146.

Bardeen, J., Thorne, K. S., and Meltzer, D.: 1966, Astrophys. J. 145, 505.

Brill, D. R. and Cohen, J. M.: 1966, Phys. Rev. 143, 1011.

Cameron, A. G. W.: 1970, this symposium, Paper 6.1, p. 323.

Canuto, V. and Chiu, H. Y.: 1968, Phys. Rev. 173, 1210.

Chandrasekhar, S.: 1964, Astrophys. J. 140, 417. 
Cocke, W. J.: 1965, Ann. Inst. Henri Poincaré, 2, No. 4, 283.

Cohen, J. M.: 1965, Summer Institute on Relativity Theory and Astrophysics (Cornell); notes appear in Lectures in Applied Math. 8: Relativity Theory and Astrophysics (ed. by J. Ehlers), A.M.S., Providence, 1967.

Cohen, J. M.: 1967, J. Math. Phys. 8, 1477.

Cohen, J. M.: 1968a, Phys. Rev. 173, 1258.

Cohen, J. M.: 1968b, J. Math. Phys. 9, 905.

Cohen, J. M.: 1968c, N. Y. Pulsar Conference, 20-21 May.

Cohen, J. M. and Brill, D. R.: 1968, Nuovo Cimento 56B, 209.

Cohen, J. M. and Cameron, A. G. W.: 1969, Nature 224, No. 5219, 566.

Cohen, J. M. and Cameron, A. G. W.: 1970, Astrophys. Space Sci. (in press).

Cohen, J. M., Langer, W., Rosen, L., and Cameron, A. G. W.: 1969a, Astrophys. Space Sci. 6, 228.

Cohen, J. M., Lapidus, A., and Cameron, A. G. W.: 1969b, Astrophys. Space Sci. 5, 113.

Cohen, J. M. and Cohen, M. D.: 1969, Phys. Teacher 7, No. 4, 241.

de la Cruz, V., Chase, J. E., and Israel, W.: 1970, Phys. Rev. Letters 24, 423.

Faulkner, J. and Gribbin, J.: 1968, Nature 217, 734.

Finzi, A. and Wolf, R. A.: 1969, Astrophys. J. Letters 155, L107.

Ginzburg, V. L. and Ozernoi, L. M.: 1965, Soviet Phys. - JETP 20, 689.

Gold, T.: 1968, Nature 218, 731.

Goldreich, P. and Julian, W. H.: 1969, Astrophys. J. 157, 869.

Gunn, J. and Ostriker, J.: 1969, Nature 221, 454.

Hartle, J. B.: 1970 , to be published.

Hartle, J. B. and Thorne, K. S.: 1968, Astrophys. J. 153, 807.

Hewish, A., Bell, S. J., Pilkington, J. D. H., Scott, P. F., Collins, R. A.: 1968, Nature 217, 709.

Landau, L. and Lifshitz, E.: 1962, Classical Theory of Fields, Addison-Wesley, Reading, Mass.

Michel, C.: 1969, Astrophys. J. 157, 1183.

Møller, C.: 1962, Theory of Relativity, Oxford Press, New York.

Skilling, J.: 1968, Nature 218, 923.

Taub, A. H.: 1962, Colloq. Intern. Centre Nat. Rech. Sci. 91, 173.

Thorne, K. S.: 1970, to be published.

Wheeler, J. A.: 1966, Annual Rev. Astron. Astrophys. 4.

Zel'dovich, Ya.: 1970, to be published.

Discussion of this paper was deferred until after the paper by Bonazzola (6.4). 